\title{
Determination of phase transformation surfaces around crack tip in shape memory alloys
}

\author{
Mohamed Rachid Laydi, Christian Lexcellent \\ Département Mécanique Appliquée, Institut FEMTO-ST, 24 rue de l'Epitaphe, 25000 BESANÇON
}

\begin{abstract}
In shape memory alloys (SMAs), a precise determination of phase transformation surfaces around the crack tip, is very important for the prediction of fracture parameters. In this work, the size of phase transformation region surrounding the tip of a edge crack is evaluated analytically. The purpose is restricted to tip without and with curvature loaded in mode I.
\end{abstract}

\section{Introduction}

Upon loading, as there is high stress concentration around the crack tip, phase transformation of edge crack, take place from austenite to martensite.

The response size must be greater for a plate without curvature at the tip because of the stress singularity in $\left(\frac{1}{\sqrt{r}}\right)$, than the plate with curvature with non singular stress state associated.

The difference between stress concentration and stress singularity can be used in order to remove the singularity at the edge of an angular defect. In order to stop the fracture increase, it is sufficient to "round" the tip by drilling circular holes.

The calculations of phase transformation region around a center crack in a SMA were first performed by Birman (1998) [1].

Freed and Banks-Sills (2007) [2] used an "HuberVon Mises" model performed by Panoskaltsis et al (2004) [3] to calculate the transformation surface under mode I loading. Recall that an Huber-Von-Mises model does not take into account the eventual asymmetry between tension and compression in the intrinsic behavior of material.

In order to include this asymmetry, Lexcellent and Thiebaud (2008) [4] multiply the equivalent HuberVon-Mises stress by a "correction function" taking into account the third invariant of stress deviator tensor.

More recently, Maletta and Furgiuele (2010) [5] used bilinear stress-strain relations with Irwing's plasticity corrected equation to calculate the extent of martensite.

In order to determine the size and the shape of the transformation zone, Baxevanis et al (2012) implement the Lagoudas SMA constitutive model in Abaqus [6].
At last, Hazar et al. (2015) [7] performed modeling of steady-state crack growth using a stationary method.

At the experimental point of view, Daly et al [8] used digital image correlation (DIC) on an edge cracked thin sheet of NiTi under mode I loading. They measure strain field around the crack tip and determine the extent of transformation along the crack.
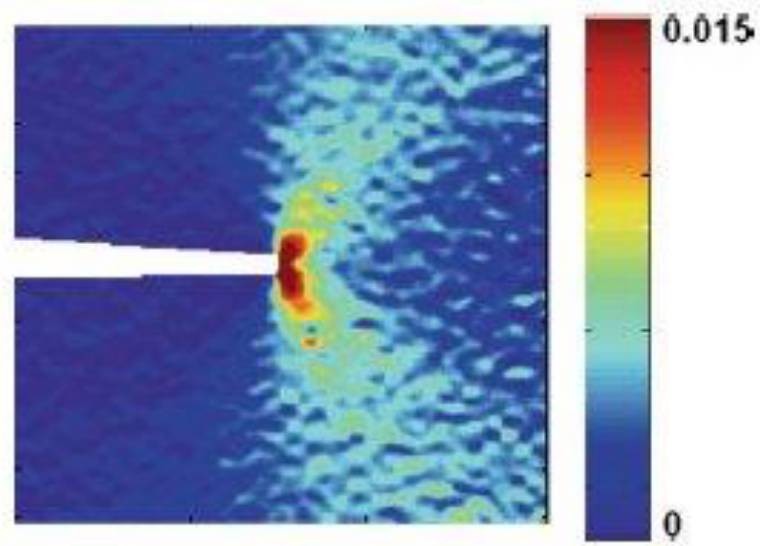

Figure 1. Measurement of $\varepsilon_{\mathrm{yy}}$ obtained by DIC at the crack tip for $\mathrm{KI}=44 \mathrm{MPa} \sqrt{m}[8]$.

Roberston et al [9] used X-ray diffraction to obtain local strain maps. They concluded that the shape was consistent with the one predicted by linear elastic fracture mechanics (LEFM).

\section{Determination of the transformation zone around the crack tip, without curvature, loaded in mode I}


The general form of the phase transformation surface equation is taken as

$$
\begin{aligned}
& F(\underline{\sigma}, T)=\bar{\sigma} f\left(y_{\sigma}\right)-\sigma_{\mathrm{c}}(T)=0 \\
& \text { where } \bar{\sigma}=\left(\frac{3}{2} \operatorname{dev} \underline{\sigma}: \operatorname{dev} \underline{\sigma}\right)^{1 / 2}
\end{aligned}
$$

is the Huber-Von-Mises equivalent stress.

$\mathrm{f}$ is some "correction function" which permits to take account the asymmetry between tension and compression.

For instance, Bouvet et al [10] chose the following expression for $\mathrm{f}$ :

and

$$
f\left(y_{\sigma}\right)=\cos \left(\frac{\cos ^{-1}\left(1-a\left(1-y_{\sigma}\right)\right)}{3}\right)
$$

$$
y_{\sigma}=\frac{27}{2} \frac{\operatorname{det}(\operatorname{dev} \underline{\sigma})}{\underline{\sigma}^{3}}
$$

One can demonstrate that the yield phase transformation surface $\mathrm{F}(\underline{\sigma}, \mathrm{T})$ is convex whatever a $\in$ $[0,1][11]$.

For mode I, under the assumption of a small scale transformation zone around the crack tip, the stress field is given by

$$
\left\{\begin{array}{l}
\sigma_{x x}^{I}(r, \theta)=\frac{K_{I}}{\sqrt{2 \pi r}} \cos \frac{\theta}{2}\left(1-\sin \frac{\theta}{2} \sin \frac{3 \theta}{2}\right) \\
\sigma_{y y}^{I}(r, \theta)=\frac{K_{I}}{\sqrt{2 \pi r}} \cos \frac{\theta}{2}\left(1+\sin \frac{\theta}{2} \sin \frac{3 \theta}{2}\right) \\
\sigma_{x y}^{I}(r, \theta)=\frac{K_{I}}{\sqrt{2 \pi r}} \cos \frac{\theta}{2} \sin \frac{\theta}{2} \cos \frac{3 \theta}{2} \\
\sigma_{x z}^{I}=\sigma_{y z}^{I}=0 \\
\sigma_{z z}^{I}= \begin{cases}0 & \text { for plane stress (CP) } \\
v\left(\sigma_{x x}+\sigma_{y y}\right) & \text { for plane strain (DP) }\end{cases}
\end{array}\right.
$$

where $r$ and $\theta$ are the crack tip polar coordinates.

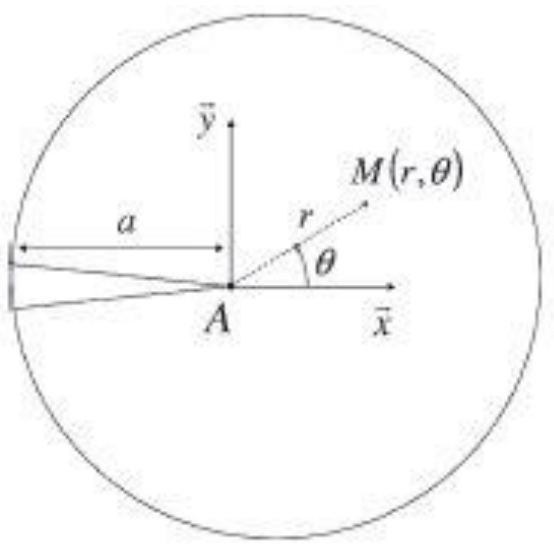

Figure 2. Reference diagramm in polar coordinates.

More over : $\quad \beta= \begin{cases}1 & \text { for plane stress } \\ 1-2 v & \text { for plane strain }\end{cases}$

with $v=0.3$. After some calculation, the yield radius value $r_{f}$ is obtained with

$$
r_{f}=\frac{1}{4 \pi}\left(\frac{K_{I}}{\sigma_{c}}\right)^{2} \mathrm{R}(\theta) \mathrm{f}^{2}\left(\mathrm{y}_{\sigma}\right)
$$

with

$$
R(\theta)=\beta^{2}(1+\cos \theta)+\frac{3}{2} \sin ^{2} \theta
$$

and

$$
\mathrm{y}_{\sigma}(\theta)=2 \sqrt{2} \beta \cos ^{3} \frac{\theta}{2} \frac{\left(9 \sin ^{2} \frac{\beta}{2}-\beta^{2}\right)}{\left(\beta^{2}(1+\cos \theta)+\frac{3}{2} \sin ^{2} \theta\right)^{3 / 2}}
$$

One can verify that $\mathrm{y}_{\sigma} \in[-1,1]$ when $\theta$ varies from 0 to $2 \pi$. Equation (7) describes the transformation zone closes to the crack tip for $\mathrm{z}=1$ (i.e. $\sigma_{\mathrm{c}}=\mathrm{b}\left(\mathrm{T}-M_{F}^{o}\right)$ ) which is the full martensite. In figures 3 and 4 , the length parameters have been normalized with respect to the quantity

$\mathrm{L}_{\mathrm{AMF}}=\frac{1}{4 M}\left[\frac{K_{I}}{b\left(T-M_{F}^{o}\right)}\right]^{2} \Rightarrow \frac{r_{f}}{L_{A M f}}=\mathrm{R}(\theta) \mathrm{f}^{2}\left(\mathrm{y}_{\sigma}(\theta)\right)$

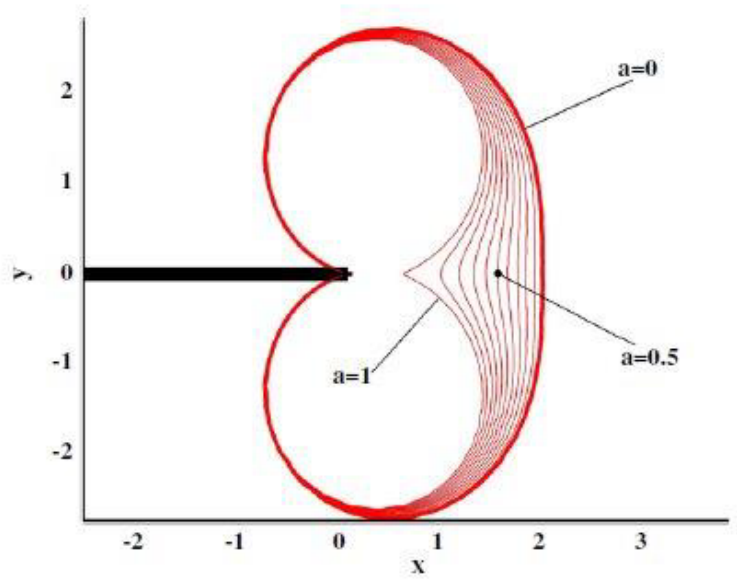

Figure 3. Normalized transformation zone value $r_{f} / L_{A M f}$ versus $\theta$ in the plane stress case, for several values of the asymmetry parameter a $(0 \leq \mathrm{a} \leq 1)$

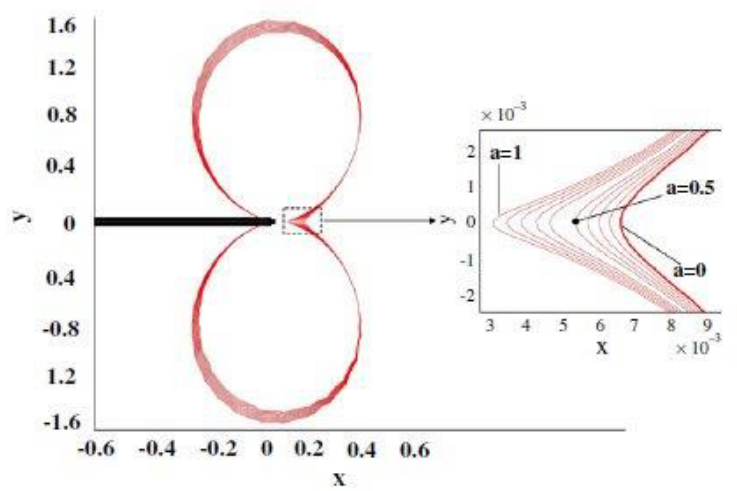

Figure 4. Normalized transformation zone value $r_{f} / L_{A M f}$ versus $\theta$ in the plane strain case, for several values of the asymmetry parameter a $(0 \leq \mathrm{a} \leq 1)$. 
About figure (3) : CP and figure (4) : DP, one can make two remarks :

- The extent of the transformation zone is clearly greater for plane stress than plane strain

- The effect of asymmetry between tensioncompression is negligible for plane strain

\section{Determination of the transformation zone around the crack tip with curvature loaded in mode I}

Starting from the classical stress field around the crack tip for mode I (without curvature radius equations (5)), Creager and Paris [12] extended the solution for finite curvature $\rho$ as :

$$
\left\{\begin{array}{c}
\sigma_{x x}=\sigma_{x x}^{I}-\frac{K_{I}}{\sqrt{2 \pi r}}\left(\frac{\rho}{2 r}\right) \cos \frac{3 \theta}{2} \\
\sigma_{y y}=\sigma_{y y}^{I}+\frac{K_{I}}{\sqrt{2 \pi r}}\left(\frac{\rho}{2 r}\right) \cos \frac{3 \theta}{2} \\
\sigma_{x y}=\sigma_{x y}^{I}-\frac{K_{I}}{\sqrt{2 \pi r}}\left(\frac{\rho}{2 r}\right) \sin \frac{3 \theta}{2} \\
\sigma_{x z}=\sigma_{x z}=0 \\
\sigma_{z z}= \begin{cases}0 & \text { for plane stress } \\
v\left(\sigma_{x x}+\sigma_{y y}\right) & \text { for plane strain }\end{cases}
\end{array}\right.
$$

Note that $r$ has a finite value on the crack tip with equation $\mathrm{r}=\frac{\rho}{2}$.

The resolution process is the same than the case of the crack tip without curvature but the calculation more complicated. They are explicated in the book of $\mathrm{C}$. Lexcellent [13].

Recall that :

$$
\left\{\begin{array}{l}
\sigma_{y z}=\sigma_{x x}=0 \\
\sigma_{z z}=0 \text { for plane stress CP } \\
\sigma_{z z}=v\left(\sigma_{x x}+\sigma_{y y}\right) \text { for plane strain DP }
\end{array}\right.
$$

For a given yield value $\sigma_{c}(T)>0$ one research the function :

$$
\mathrm{r}: \theta \in[0,2 \pi] \rightarrow \mathrm{r}(\theta) \geq \frac{1}{2} \rho>0
$$

solution of the non-linear equation :

$$
\mathrm{F}(\sigma)=\bar{\sigma} \mathrm{f}\left(\mathrm{y}_{\sigma}\right)-\sigma_{\mathrm{c}}(\mathrm{T})=0
$$

If one examines the particular case of Huber-Von Mises e.g. with $f=1$

$$
\bar{\sigma}=\sigma_{\mathrm{c}}
$$

an auxiliary variable is introduced :

$$
\begin{gathered}
\mathrm{z}=\frac{\rho}{2 r} \\
\mathrm{z}=\psi_{\mathrm{p}}(\chi)=\left(\sqrt{\chi^{2}+p^{3}}+\chi\right)^{1 / 3}-\left(\sqrt{\chi^{2}+p^{3}}+\chi\right)^{1 / 3}
\end{gathered}
$$

and hence shown that :

$$
\mathrm{r}=\frac{1}{2} \frac{\rho}{\psi_{p}(\chi)}
$$

is solution of the Huber-Von Mises equation if and only if :

$$
\chi \leq \frac{1}{2}
$$

In the general case, $\sigma_{\mathrm{c}}$ is substituted by $\frac{\sigma_{c}}{f\left(y_{\sigma}\right)}$ or by $\frac{\sigma_{c}}{f\left(y_{\Sigma}\right)}$ because :

$$
\begin{aligned}
& \bar{\sigma}=\frac{K_{I}}{\sqrt{\pi \rho}} z^{\frac{1}{2}} \bar{\Sigma} \text { and } \mathrm{y}_{\sigma}=\mathrm{y}_{\Sigma} \\
& \text { e.g. : } \frac{K_{I}}{\sqrt{\pi \rho}} z^{\frac{1}{2}} \bar{\Sigma}=\bar{\sigma}=\frac{\sigma_{c}}{f\left(y_{\Sigma}\right)}
\end{aligned}
$$

More precisely, there is equivalences

$\mathrm{G}(\sigma)=\bar{\sigma} f\left(y_{\Sigma}\right)=\sigma_{c} \Leftrightarrow \sqrt{z} G(\Sigma)=2 \kappa \chi^{\frac{1}{2}} \Leftrightarrow z=$ $\psi_{p}\left(\chi \alpha_{\theta}(z)\right)$

where $\alpha_{\theta}$ is a continuous function of $\mathrm{z}$ and $\theta$ defined by $\alpha_{\theta}(\mathrm{z}) \equiv\left|\mathrm{f}\left(\mathrm{y}_{\Sigma}\right)\right|^{-2}$

Its expression is easily obtained by combining the value of $\left|\mathrm{S}_{\Sigma}\right|$ with $\operatorname{det}\left(\mathrm{S}_{\Sigma}\right)=\frac{2 \beta}{3} \cos \frac{\theta}{2}\left(z^{2}+\frac{1}{4} \sin ^{2} \theta\right)$.

Moreover, we have the following framing :

$$
\frac{1}{8} \eta^{3} \leq \chi \alpha_{\theta}(z) \leq \frac{1}{2} \eta^{3} \text { where } \eta=\left(6 \chi|\mathrm{f}(0)|^{-2}\right)^{1 / 3}
$$

This result is the direct consequence of the G convexity (Laydi et Lexcellent [11]) which imposes to $f$ the necessary following condition :

$$
3^{-\frac{1}{2}} f(0) \leq f(y) \leq 2.3^{-\frac{1}{2}} f(0) \quad \forall \mathrm{y} \in[-1,1]
$$

The problem resolution consists to find $\hat{z}$ solution of one following equation :

$$
\varphi_{\mathrm{p}}(\hat{z})=\chi \alpha_{\theta}(\hat{z}) \Leftrightarrow \hat{z}=\psi_{\mathrm{p}}\left(\chi \alpha_{\theta}(\hat{z})\right)
$$

Let pose :

$$
\xi \equiv \psi_{\frac{4}{27}}\left(\frac{1}{8} \eta^{3}\right)
$$

One can demonstrate the following theorem.

Let $\eta \in] 0,1]$. Then, the yield surface equation admits at least one solution $r=\frac{\rho}{2 z}$ such that :

$$
\xi \leq \psi_{p}\left(\frac{1}{8} \eta^{3}\right) \leq z \leq \psi_{p}\left(\frac{1}{2} \eta^{3}\right) \leq \eta \forall \theta
$$

Moreover, if :

$$
\eta \leq 0.34
$$




\section{MATEC Web of Conferences}

Then, the problem solution is unic

\section{Applications}

Let consider the following physical parameters :

$v=0.3, \rho=0.5 \mathrm{~mm}, \mathrm{~K}_{\mathrm{I}}=50 \mathrm{MPa} \sqrt{m}$, phase transformation initiation stress $\sigma_{8}=30 \mathrm{MPa}$.

phase transformation end stress $\sigma_{\mathrm{f}}=60 \mathrm{MPa}$.

\section{Application I}

$$
\begin{aligned}
& \text { Let } \mathrm{f}(\mathrm{y})=1+\mathrm{by}, \mathrm{b} \in\left[0, \frac{1}{8}\right] \\
& \qquad \begin{array}{l}
\left.\eta=\left.(6 \chi \mid f(0))\right|^{-2}\right)^{\frac{1}{3}}=\left(\pi \rho\left(\frac{\sigma_{c}}{K_{I}}\right)^{2}|f(0)|^{-2}\right)^{\frac{1}{3}} \\
=\left\{\begin{array}{c}
0.08 \text { transformation beginning } \\
.013 \text { transformation end }
\end{array}\right\}<0.34
\end{array}
\end{aligned}
$$
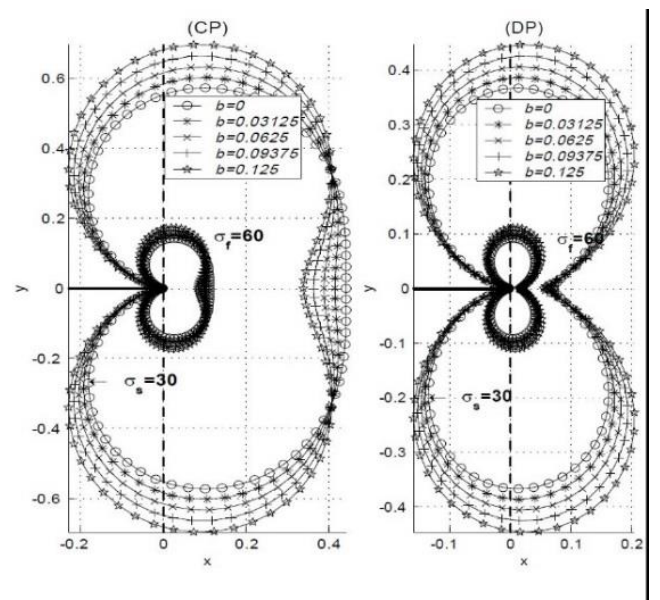

Figure 5. Cracked plate with mode I curvature radius. (b : parameter of $\mathrm{f}$ function).

\section{Application II}

Let $\mathrm{f}(\mathrm{y})=\cos \left(\frac{1}{3} \operatorname{arcos}(1-a(1-y))\right), \forall \mathrm{a} \in[0,1]$.
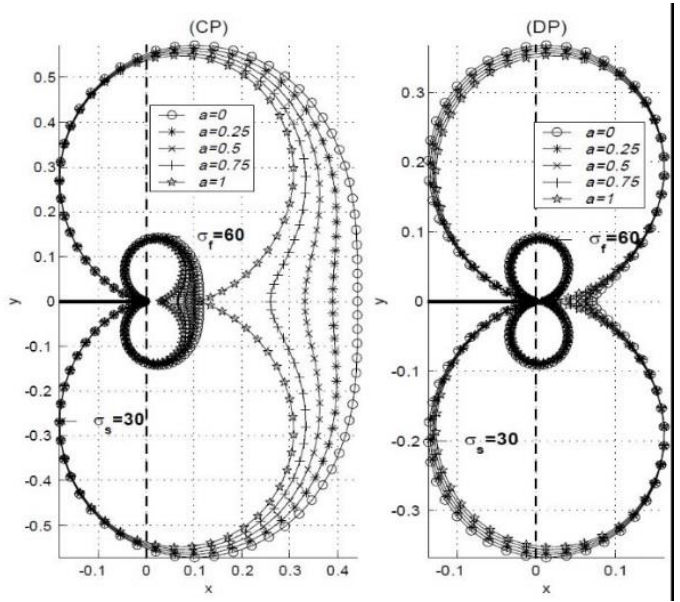

Figure 6. Cracked plate with mode I curvature radius. (a : parameter of $\mathrm{f}$ function).

\section{Conclusion}

One can see on figures 3 and 4 on one part and figures 5 , 6 on an another part that the size of phase transformation zone is greater around crack tip without curvature than with curvature.

The experimental tests are performed with curvature for technological reasons.

For instance, the figure 7 shows a plate with curvature radius under mechanical loading.

The shape of the transformation zone is the same as predicted in plane stress conditions.

In a logical way, one would examine the mode II loading. In spite of the fact that the stress field is given a new time by Creager and Paris [12], the resolution seems more complicated.

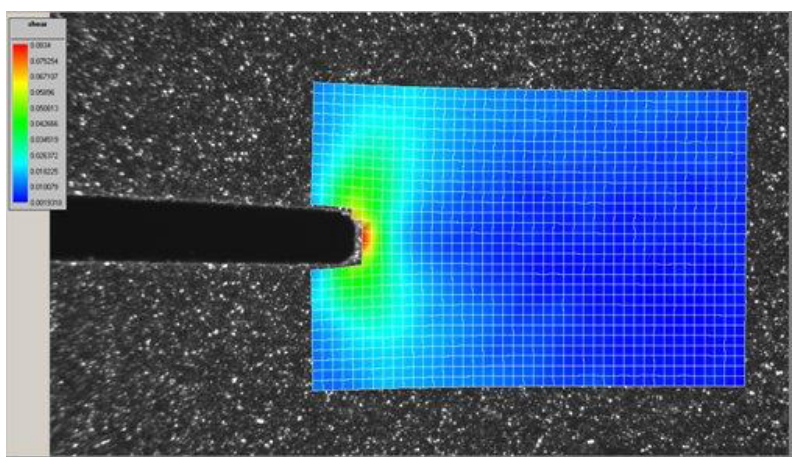

Figure 7. Overall photo of a plate with a curvature radius under mechanical loading [13]

\section{References}

1. V. Birman, Smart Mater. Struct., 7, 433 (1990)

2. Y. Freed, L. Banks-Sill, J. Mech. Solids, 55, 2157 (2007)

3. V.P. Panoskaltsis, S. Bahuguna, D. Soldatos, Int. J. Non. Linear. Med., 39, 709 (2004)

4. C. Lexcellent, F. Thiebaud, Scr. Mater., 59, 321 (2008)

5. C. Maletta, F. Furgiuele, Acta Mater., 58, 92 (2010)

6. T. Baxevanis, Y. Chemisky, D.C. Lagoudas, Smart Mater. Struct., 21, 94 (2012)

7. S. Hazar, W. Zaki, Z. Moumni, G. Anlas, Int. J. Plast., 67, 26 (2015)

8. S. Daly, A. Miller, G. Ravichandran, K. Bhattacharya, Acta Mater., 55, 6322 (2007)

9. S.W. Robertson, A. Mehta, A.R. Pelton, R.O Ritchie, Acta Mater., 55, 6198 (2007)

10. C. Bouvet, S. Calloch, C. Lexcellent, Eur. J. Eng. Mater. Technol., 124, 665 (2002)

11. M.R. Laydi, C. Lexcellent, Arch. Ration. Mech. Anal., 204, 977 (2012)

12. M. Greager, P. Paris, Int. J. of Fracture, 3, 247 (1967)

13. C. Lexcellent, "Shape-memory alloys handbook", ISTE-WILEY, chap. 10, pp. 322-326 (2013) 